\title{
Estrategias de intervención en la prevención de comportamiento suicida en pacientes con trastorno por consumo de sustancias en tiempos de COVID-19
}

\section{Intervention strategies in the prevention of suicidal behavior in substance use disorders patients in times of COVID-19}

\author{
Ashikan Espandian*, Gerardo Flórez******, Luisa F. Peleteiro****, María Tajes****, \\ PILAR A. SÁIZ***,*****,******,*******, Rocío VILla******,*******, \\ JuLio BobES $* * *, * * * * *, * * * * * *, * * * * * * *$. \\ * Servicio de Psiquiatría. Complejo Hospitalario del Bierzo. Ponferrada. \\ ** Unidad de Conductas Adictivas, Complejo Hospitalario Universitario de Ourense, España. \\ *** Centro de Investigación Biomédica en Red de Salud Mental (CIBERSAM), España. \\ **** Servizo de Saúde Mental. Dirección de Asistencia Sanitaria SERGAS. \\ ****** Área de Psiquiatría, Universidad de Oviedo, España. \\ ******* Servicio de Salud del Principado de Asturias (SESPA), España. \\ ******* Instituto de Investigación Sanitaria del Principado de Asturias (ISPA), España.
}

$\mathrm{E}$ 1 suicidio representa un grave problema para la salud pública, tanto por su elevada prevalencia en el momento actual como por la falta de implementación de adecuadas estrategias preventivas en una buena parte de países, incluida España (Sáiz y Bobes, 2014).

Se estima que el número de tentativas suicidas (TS) es, aproximadamente, unas 10-20 veces superior al de los suicidios consumados. Sin embargo, la magnitud real del problema está infraestimada por la tendencia a la subnotificación que se realiza en la mayoría de los países del mundo (Giner y Guija, 2014).

Hasta la fecha, la historia previa de TS es el mejor predictor de posterior suicidio consumado (Wang, Huang, Lee, Wu y Chen, 2014). La existencia de trastornos mentales es otro de los principales factores de riesgo, siendo el trastorno depresivo uni y bipolar y los trastornos por consumo de alcohol los más prevalentes en relación con el comportamiento suicida (Gómez-Durán, Forti-Buratti, Gutiérrez-López, Belmonte-Ibáñez y Martín-Fumadó, 2016; Zalsman et al., 2016).

La entrevista clínica es la herramienta principal para obtener una impresión clínica sobre el riesgo suicida presente en un determinado momento en un paciente, tal y como recomiendan las guías clínicas más actualizadas, que indican que los instrumentos de screening no disponen de suficiente capacidad predictiva (Grupo de Trabajo de revisión de la Guía de Práctica Clínica de prevención y tratamiento de la conducta suicida, 2020; Jacobs et al., 2010; Inagaki, Kawashima, Yonemoto y Yamada, 2019; Mann et al., 2005).

El objetivo de la entrevista clínica es detectar los factores de riesgo suicida, a la vez que se contrastan con los factores protectores que estén operando en el momento de la valoración. El conocimiento claro y su identificación suponen un punto fundamental en relación con la determinación del nivel de riesgo, recalcando que algunos tienen un peso más específico que otros y que su combinación aumenta el riesgo de forma considerable (Hawton y van Heeringen, 2009; Kraemer y Clarke, 1990).

Como herramienta de consulta y apoyo al clínico, se presentan los principales factores de riesgo en la tabla 1 y los factores de protección en la tabla 2.

\section{Consumo de sustancias y comportamiento suicida}

El suicidio representa la principal causa de muerte en persona con trastorno por consumo de sustancias (Wilcox, 
Estrategias de intervención en la prevención de comportamiento suicida en pacientes con trastorno por consumo de sustancias en tiempos de COVID-19

Tabla 1. Principales factores de riesgo asociados al comportamiento suicida.

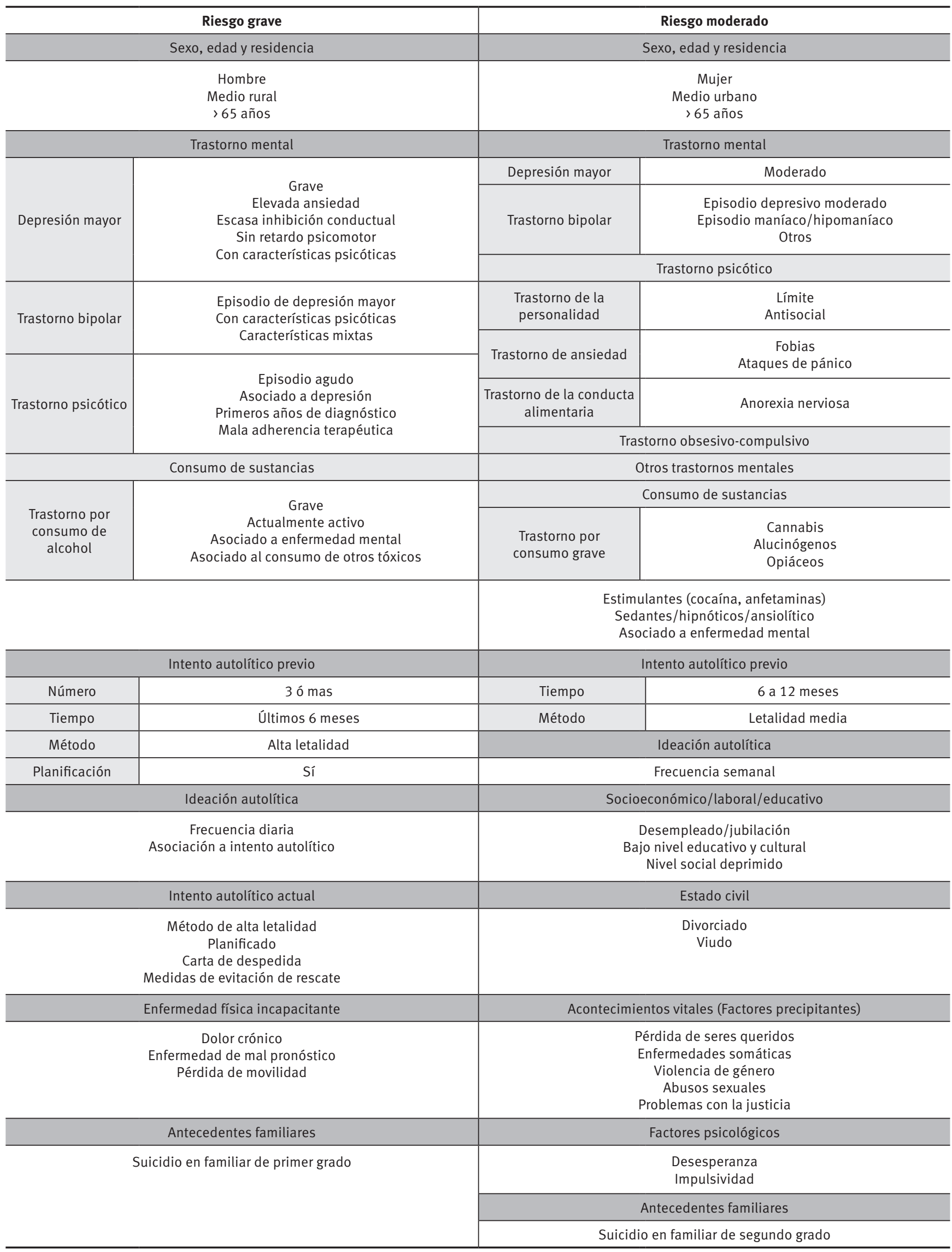


Tabla 2. Principales factores de protección frente al comportamiento suicida.

\begin{tabular}{ll}
\hline Personales o propios del individuo & Sociales y medioambientales \\
\hline - Actitudes y valores del propio individuo & $\bullet$ Tener un buen apoyo familiar y social \\
- Habilidades en la resolución de conflictos o problemas & $\bullet$ Integración social \\
- Presencia flexibilidad cognitiva & $\bullet$ Accesibilidad a los dispositivos de salud \\
- Tener confianza en uno mismo & $\bullet$ Tratamiento integral, permanente y a largo plazo a pacientes con trastornos mentales \\
- Habilidades para las relaciones personales e interpersonales & $\bullet$ Control a la hora de adquirir armas o medicaciones potencialmente letales \\
- Creencias religiosas que desaprueben el suicidio & \\
\hline Autocontrol de la impulsividad y manejo de la ira & \\
\hline
\end{tabular}

Conner y Caine, 2004). Se estima que el riesgo de muerte por suicidio con respecto a la población general aumenta 10 veces para el trastorno por consumo de alcohol y 14 veces para el adicto a otras sustancias (Yuodelis-Flores y Ries, 2015).

El 3,4\% de los hombres y el 4,4\% de las mujeres con consumo de sustancias realizan una TS en los 30 días previos a la incorporación en un programa de tratamiento (Tiet, Ilgen, Byrnes y Moos, 2006). La presencia de una elevada prevalencia tanto de ideación suicida como de TS al inicio del tratamiento parece estar relacionada con periodos donde el consumo se encuentra fuera de control, con la presencia de problemas en el entorno (laboral, marital, legal, etc.) y con el inicio o reagudización de la enfermedad mental (Ross et al., 2005).

Con relación al sexo, se observa un riesgo mayor tanto para la TS como para el suicidio consumado en el sexo femenino, a diferencia del patrón que se establece en la población general donde el suicidio predomina en el hombre de forma destacada. Las comorbilidades psiquiátricas y el desajuste social que conlleva el consumo de sustancias son considerados factores relevantes asociados al incremento del riesgo en mujeres (Wilcox et al., 2004).

Los trastornos relacionados con el consumo de alcohol tienen un papel destacado en el comportamiento suicida. No solo como factor de riesgo, sino también como un factor precipitante, debido a la desinhibición y disfunción ejecutiva que produce la intoxicación alcohólica (World Report on Violence and Health, 2002). Se estima que en el $37 \%$ de los suicidios consumados se había producido un consumo de alcohol previo, dicha tasa se eleva a un $40 \%$ en los no letales (Wilcox et al., 2004). Siendo el incremento del riesgo 15 veces mayor con respecto a la población general (Beautrais, Collings, Ehrhardt y Henare, 2005).

Los consumos de otras sustancias también incrementan el riesgo de comportamiento suicida frente a la población general. Se estima que el riesgo de suicidio es de 14 a 17 veces mayor que el de la población general, estando principalmente en relación con la afectación cognitiva, el desajuste social y la comorbilidad psiquiátrica (Price, Hemmingsson, Lewis, Zammit y Allebeck, 2009; Tiet et al., 2006; Wilcox et al., 2004).
Por otra parte, el policonsumo incrementa, como es lógico, el riesgo suicida. El 58\% de los pacientes policonsumidores tienen antecedentes de tentativas suicidas y su riesgo es 17 veces mayor que el de la población general; además se incrementa la letalidad de la sobredosis, método suicida más frecuente en la población adicta (Wilcox et al., 2004).

La gravedad del consumo, así como el número de sustancias que se consumen representan un factor de riesgo, con una relación con las TS, de mayor impacto que el tipo de sustancia consumida (Ilgen, Harris, Moos y Tiet, 2007).

En relación a la comorbilidad psiquiátrica, el riesgo aumenta de forma considerable cuando se asocia otro trastorno mental al consumo de sustancias. Destaca el trastorno depresivo como la asociación más lesiva, seguido del trastorno de la personalidad y el trastorno en el control de los impulsos (Artenie et al., 2015; Wilcox et al. 2004).

\section{Intervención en el paciente con trastorno por consumo de sustancias como método de prevención}

Las evidencias actuales ponen claramente de manifiesto la elevada prevalencia del comportamiento suicida en los pacientes con consumo de sustancias. Las estrategias de prevención representan un desafío que se incrementa en este perfil de pacientes, siendo una población de riesgo en la que todavía no está clara la estrategia que emplear para poder reducir la tasa de muertes por suicidio (Goldstone, Bantjes y Dannatt, 2018).

De las estrategias de prevención de que se dispone hoy en día con relación a la prevención del comportamiento suicida, son pocas las que gozan de una evidencia suficiente para asegurar que reducen la incidencia de suicidio en la población general. En el año 2017, se publican las principales estrategias que han demostrado evidencia en la prevención a nivel de la salud pública como la atención sanitaria (Zalsman et al., 2017).

Se está trabajando en la implementación de estrategias de prevención en los diferentes grupos de riesgo, pero se carece de estudios de intervenciones desarrolladas en la población de pacientes consumidores de sustancias 
(Goldstone et al., 2018). Sería de gran utilidad disponer de información que nos pueda indicar qué medidas de intervención son las óptimas para aplicar en esta población; o reafirmar que las estrategias utilizadas en la población general presentan la misma eficacia en este perfil de pacientes de riesgo.

Hoy por hoy se han publicado los resultados de dos proyectos piloto que investigan la eficacia de dos intervenciones diferentes en población consumidora de sustancias (Esposito-Smythers, Spirito, Uth y LaChance, 2006; Voss et al., 2013). Dichos resultados ponen de manifiesto que las intervenciones aportan beneficios en este perfil de pacientes, fundamentalmente mejorando la adherencia, reduciendo la ideación suicida y facilitando una mayor capacidad de gestión de los momentos de ansiedad con un incremento de la capacidad de solicitar ayuda. Los programas aportan una gran cantidad de información al paciente referente a la asociación del consumo con los comportamientos suicidas, lo que les lleva a una mejor actitud y manejo de la situación. Lamentablemente, no se observa utilidad de dichos programas en la prevención de la repetición de la TS.

Por ello, nos encontramos con datos favorables que nos orientan hacia un modelo factible y viable de aplicar como estrategias de prevención, estando altamente indicadas en este perfil de pacientes. Por otro lado, conviene remarcar que dichos resultados tienen que ser interpretados con precaución, ya que representan estudios piloto, en los cuales existen múltiples limitaciones, como el reducido tamaño muestral, el empleo de pruebas psicométricas poco específicas e inadecuadas que nos plantean su mejoría en futuros estudios y la carencia de un grupo control con el que se pueda comparar. Los escasos estudios referentes al tema dejan una puerta abierta hacia el análisis y la búsqueda de nuevas estrategias en este perfil de pacientes.

Las estrategias de prevención a tres niveles (universal, selectiva e indicada) obtienen grandes resultados cuando se combinan, estando posicionados los pacientes con diagnóstico de consumo de sustancias en el grupo de población de riesgo y de intervención indicada. Sería de gran utilidad disponer de recursos para su utilización en la mejora del tratamiento estándar en este grupo de pacientes. Principalmente en la formación de los profesionales sanitarios en los tres niveles de intervención, siendo estos: el tratamiento del consumo, de la comorbilidad psiquiátrica y la prevención del comportamiento suicida. A través de este tratamiento se puede brindar una intervención más específica y distinta a la genérica (Goldstone et al., 2018).

Una parte importante del personal que ejerce en las unidades de conductas adictivas reconoce su falta de formación con respecto a la intervención en los pacientes de riesgo suicida. Una formación adecuada aportaría conocimientos y confianza en el terapeuta en el manejo, lo que conlleva una mejora en la adherencia terapéutica.
Habiéndose evidenciado que los años de experiencia ejerciendo en la unidad de tratamiento de conductas adictivas no suponen una equivalencia a la capacidad en el manejo del paciente de riesgo suicida (Fruhbauerova y Comtois, 2019).

\section{Entrevista e intervención en pacientes con riesgo suicida y trastorno por consumo de sustancias}

Toda la intervención debe estar basada en los siguientes principios psicoterapéuticos: empatía, colaboración y honestidad. Es decir, el profesional sanitario no se posiciona como guardián de la integridad física del paciente, sino como un profesional preocupado por su sufrimiento, que quiere que el paciente siga viviendo, pero entiende sus dificultades y respeta el punto de vista del paciente y sus decisiones, ofreciéndole su ayuda para mejorar la situación y superar las crisis. El profesional sanitario debe recordar que en la mayoría de los casos existirá una ambivalencia entre la vida y la muerte que hay que explorar para conocer y poder intervenir en los factores que determinan esa ambivalencia.

Es conveniente trabajar con periodos de tiempo claramente establecidos. Es fundamental que el terapeuta informe al paciente sobre el marco legal y asistencial en el que se desarrolla la intervención y cuáles son sus obligaciones en este marco.

A continuación, se exponen estrategias a utilizar durante la intervención psicoterapéutica:

\section{Balance decisional}

Una actividad de gran utilidad a realizar con el paciente es un balance decisional sobre las razones para vivir versus las razones para morir. Debe realizarse de forma colaborativa y honesta sin que el terapeuta se posicione de forma evidente a favor de las razones para vivir.

\section{Plan estabilización - intervención en crisis}

El plan de estabilización - intervención en crisis se elabora en consulta con el paciente y este debe llevarlo siempre consigo, en forma de tarjeta o en el teléfono móvil. Los elementos del plan deben ser sencillos y de fácil aplicación. Por lo menos, debe incluir los siguientes elementos:

- Señales de aviso para activar el plan.

- Maneras de reducir el acceso a métodos letales.

- Actividades de distracción y autorregulación para un momento de crisis.

- Personas con las que contactar durante un momento de crisis: deben estar disponibles y saber que se les ha asignado este papel para poder responder de forma empática a la demanda del paciente.

- Teléfonos de emergencia con los que contactar. 


\section{Incrementar las actividades que impliquen experimentar emociones positivas}

Incrementar el nivel de actividad y orientarlo hacia experiencias emocionalmente positivas es una técnica conductual fundamental a realizar con este tipo de pacientes.

\section{Mejorar las estrategias de regulación emocional}

Entrenar al paciente en técnicas de relajación, meditación/mindfulness, imaginería, y promover estrategias positivas que el paciente ya utilice para autorregularse emocionalmente es una tarea de gran importancia.

\section{Incrementar el apoyo social del paciente}

Elaborar una lista de apoyos, mayores o menores, fuera del marco terapéutico es otra estrategia muy recomendable.

\section{Incrementar el apoyo de otros servicios sanitarios y sociales mejorando la adherencia a los mismos}

Los factores de riesgo mencionados previamente pueden ser trabajados y atenuados por otros servicios sanitarios y sociales. Debe establecerse colaborativamente con el paciente una lista de posibles recursos de ayuda y plantear estrategias para mejorar la adherencia a dichos recursos.

\section{Mejorar la capacidad de manejo de pensamientos o ideas problemáticas}

Preparar notas o mensajes en el teléfono móvil con pensamientos que contrarresten la ideación suicida del paciente es de gran utilidad. Deben ser sencillos, de fácil acceso.

\section{Impacto de la pandemia COVID-19 en el consumo de sustancias}

Actualmente nos encontramos en una situación de pandemia por el COVID-19. Múltiples publicaciones realizan una previsión negativa y advierten a las autoridades sanitarias del posible incremento tanto de las tasas de TS como de suicidios consumados debido al incremento de los factores de riesgo como el desempleo, la crisis económica, los conflictos sociales, el aumento de los niveles de ansiedad y depresión, la reticencia para la búsqueda de ayuda en los centros de salud en cuanto a la dificultad para acceder a ellos, el incremento en el consumo de alcohol y finalmente la facilidad de acceso a métodos letales (lejía, desinfectantes) (Gunnell et al., 2020).

Datos obtenidos en los desastres naturales y pandemias previas, ponen de manifiesto una reducción de la tasa de suicidio momentáneo para un incremento inmediato tras el suceso, lo que apoya las previsiones y advertencias realizadas (Wasserman, Iosue, Wuestefeld y Carli, 2020).

La población con trastorno por consumo de sustancias representa un grupo vulnerable y de riesgo de contaminación por las condiciones clínicas, psicopatológicas y psico- sociales (Ornell et al., 2020). Se prevé que la cuarentena y el aislamiento social pudieran tener un efecto negativo, en cuanto al incremento en el consumo y a una mayor tasa de recaídas en individuos con largos periodos de abstinencia, estando principalmente enfocados al consumo de alcohol (Kahil et al., 2021). Por otra parte, existen datos que ponen de manifiesto como el consumo de alcohol y/o tabaco han sido estrategias utilizadas por la población general para tratar de afrontar las consecuencias derivadas de la pandemia (Martínez-Cao et al., 2021).

Existe una interacción entre la COVID-19 y las adicciones, de forma bidireccional, que conlleva una amenaza y un mayor impacto para la salud pública. Los organismos gubernamentales y legislativos deben actuar para garantizar la seguridad social en este grupo de pacientes, manteniendo la disponibilidad y acceso a todos los dispositivos de tratamiento, asociado a un aporte de información clara, accesible y de fácil comprensión en relación con las medidas de prevención contra el COVID-19 en estos grupos de pacientes (Dubey et al., 2020; García-Álvarez, Fuente-Tomás, Sáiz, García-Portilla y Bobes, 2020; Mallet, Dubertret y Le Strat, 2021). Se recomienda de forma prioritaria la necesidad de fortalecer la salud mental de cara al manejo de las secuelas que ha dejado la pandemia.

\section{Conclusiones}

Se trabaja en la prevención de la TS en la población general y en los pacientes de salud mental, pero escaso esfuerzo se promueve en la población con trastorno por consumo de sustancias. Las evidencias dejan clara la elevada prevalencia del comportamiento suicida en dichos pacientes, siendo considerado el consumo de sustancias una señal de alarma indirecta, que conlleva al seguimiento del paciente.

Por ello se recomienda la creación de protocolos de intervención en los pacientes que acuden a la unidad asistencial, iniciando un screening sistematizado de valoración del riesgo de comportamiento suicida y el desarrollo de estrategias de intervención en dichos pacientes, con el fin de conseguir tanto la reducción de las tasas de TS como de suicidio consumado, y destacando y valorando principalmente el nivel de la exclusión social, las comorbilidades psiquiátricas y los niveles de desesperanza que presenta el paciente.

Las intervenciones terapéuticas generales para el tratamiento del comportamiento suicida podrían ser aplicables en esta población, aunque la investigación hasta la fecha ha sido escasa a la hora de probar su efectividad. Se recomienda que el personal sanitario que atiende a pacientes con trastornos por consumo de sustancias esté familiarizado y posea la capacidad de identificación de los pacientes de riesgo, siendo fundamental detectar los factores de riesgo y signos de alerta en los pacientes a los que tratan. 
Conviene recalcar la especial importancia de incidir en el tratamiento de la comorbilidad psiquiátrica como instrumento terapéutico para reducir el riesgo suicida en estos pacientes.

Con esto no se pretende restar importancia a las Unidades de Intervención sobre los Comportamientos Suicidas, altamente necesarias, sino resaltar y destacar el aporte fundamental que desde las Unidades de Tratamiento de las Adicciones se puede realizar para reducir la prevalencia de estas.

\section{Reconocimientos}

Los autores agradecen el apoyo del Gobierno del Principado de Asturias PCTI 2018-2022 IDI/2018/235, CIBERSAM y Fondos Europeos de Desarrollo Regional (FEDER).

\section{Conflicto de intereses}

Los autores declaran que no existe conflicto de interés para el presente trabajo.

\section{Referencias}

Artenie, A. A., Bruneau, J., Zang, G., Lespérance, F., Renaud, J., Tremblay, J. y Jutras-Aswad, D. (2015). Associations of substance use patterns with attempted suicide among persons who inject drugs: Can distinct use patterns play a role? Drug and Alcohol Dependence, 147, 208214. doi:10.1016/j.drugalcdep.2014.11.011.

Beautrais, A. L., Collings, S. C. D., Ehrhardt, P. y Henare, K. (2005). Suicide Prevention: A review of evidence of risk and protective factors, and points of effective intervention. Wellington: Ministry of Health.

Dubey, M. J., Ghosh, R., Chatterjee, S., Biswas, P., Chatterjee, S. y Dubey, S. (2020). COVID-19 and addiction. Diabetes $\mathcal{E}$ Metabolic Syndrome, 14, 817-823. doi:10.1016/j. dsx.2020.06.008.

Esposito-Smythers, C., Spirito, A., Uth, R. y LaChance, H. (2006). Cognitive behavioral treatment for suicidal alcohol abusing adolescents: Development and pilot testing. American Journal on Addictions, 15, 126-130.

Fruhbauerova, M. y Comtois, K. A. (2019). Addiction counselors and suicide: Education and experience do not improve suicide knowledge, beliefs, or confidence in treating suicidal clients. Journal of Substance Abuse Treatment, 106, 29-34. doi:10.1016/j.jsat.2019.08.012.

García-Álvarez, L., Fuente-Tomás, L. D. la, Sáiz, P. A., García-Portilla, M. P. y Bobes, J. (2020). Will changes in alcohol and tobacco use be seen during the COVID-19 lockdown? Adicciones, 32, 85-89. doi:10.20882/adicciones. 1546 .

Giner, L. y Guija, J. A. (2014). Número de suicidios en España: Diferencias entre los datos del Instituto Nacional de Estadística y los aportados por los Institutos de Medicina Legal. Revista de Psiquiatría y Salud Mental, 7, 139146. doi:10.1016/j.rpsm.2014.01.002.

Goldstone, D., Bantjes, J. y Dannatt, L. (2018). Mental health care providers' suggestions for suicide prevention among people with substance use disorders in South Africa: A qualitative study. Substance Abuse Treatment, Prevention, and Policy, 13, 47. doi:10.1186/s13011-0180185-y.

Gómez-Durán, E. L., Forti-Buratti, M. A., Gutiérrez-López, B., Belmonte-Ibáñez, A. y Martín-Fumadó, C. (2016). Trastornos psiquiátricos en los casos de suicidio consumado en un área hospitalaria entre 2007-2010. Psiquiatría y Salud Mental, 9, 31-38. doi:10.1016/j. rpsm.2014.02.001.

Grupo de Trabajo de revisión de la Guía de Práctica Clínica de prevención y tratamiento de la conducta suicida (2020). Revisión de la Guía de Práctica Clínica de Prevención y Tratamiento de la Conducta Suicida (2012) del Programa de GPC en el SNS. Ministerio de Sanidad. Agencia Gallega para la Gestión del Conocimiento en Salud (ACIS), Unidad de Asesoramiento Científico-Técnico, Avalia-t. Guías de Práctica Clínica en el SNS.

Gunnell, D., Appleby, L., Arensman, E., Hawton, K., John, A., Kapur, N. y COVID-19 Suicide Prevention Research Collaboration (2020). Suicide risk and prevention during the COVID-19 pandemic. Lancet Psychiatry, 7, 468471. doi:10.1016/S2215-0366(20)30171-1.

Hawton, K. y van Heeringen, K. (2009). Suicide. Lancet, 373, 1372-1381. doi:10.1016/S0140-6736(09)60372-X.

Ilgen, M. A., Harris, A. H. S., Moos, R. H. y Tiet, Q. Q. (2007). Predictors of a suicide attempt one year after entry into substance use disorder treatment. Alcoholism, Clinical and Experimental Research, 31, 635-642. doi:10.1111/ j.1530-0277.2007.00348.x.

Inagaki, M., Kawashima, Y., Yonemoto, N. y Yamada, M. (2019). Active contact and follow-up interventions to prevent repeat suicide attempts during high-risk periods among patients admitted to emergency departments for suicidal behavior: A systematic review and meta- analysis. BMC Psychiatry, 19, 44. doi:10.1186/s12888-019-2017-7.

Jacobs, D. G., Baldessarini, R. J., Conwell, Y., Fawcett, J. A., Horton, L., Meltzer, H.,... Simon, R. I. (2010). Assessment and treatment of patients with suicidal behaviors. APA Practice Guidelines.1-183.

Kahil, K., Cheaito, M. A., El Hayek, R., Nofal, M., El Halabi, S., Kudva, K. G.,... El Hayek, S. (2021). Suicide during COVID-19 and other major international respiratory outbreaks: A systematic review. Asian Journal of Psychiatry, 56, 102509. doi:10.1016/j.ajp.2020.102509.

Kraemer, G. W. y Clarke, A. S. (1990). The behavioral neurobiology of self-injurious behavior in rhesus monkeys. Progress in Neuro-Psychopharmacology E Biological Psychiatry, 14, 141-168. doi:10.1016/0278-5846(90)90092-U. 
Mallet, J., Dubertret, C. y Le Strat, Y. (2021). Addictions in the COVID-19 era: Current evidence, future perspectives a comprehensive review. Progress in Neuro-psychopharmacology Eं Biological Psychiatry, 106, 110070. doi:10.1016/j. pnpbp.2020.110070.

Mann, J. J., Apter, A., Bertolote, J., Beautrais, A., Currier, D., Haas, A.,... Hendin, H. (2005). Suicide prevention strategies: A systematic review. JAMA, 294, 2064-2074. doi:10.1001/jama.294.16.2064.

Martínez-Cao, C., de la Fuente-Tomás, L., Menéndez-Miranda, I., Velasco, Á., Zurrón-Madera, P., García-Álvarez, L.,... Bobes, J. (2021). Factors associated with alcohol and tobacco consumption as a coping strategy to deal with the coronavirus disease (COVID-19) pandemic and lockdown in Spain. Addictive Behaviors, 121, 107003. doi:10.1016/j.addbeh.2021.107003.

Ornell, F., Moura, H. F., Scherer, J. N., Pechansky, F., Kessler, F. y von Diemen, L. (2020). The COVID-19 pandemic and its impact on substance use: Implications for prevention and treatment. Psychiatry Research, 289, 113096. doi:10.1016/j.psychres.2020.113096.

Price, C., Hemmingsson, T., Lewis, G., Zammit, S. y Allebeck, P. (2009). Cannabis and suicide: Longitudinal study. British Journal of Psychiatry, 195, 492-497. doi:10.1192/bjp.bp.109.065227.

Ross, J., Teesson, M., Darke, S., Lynskey, M., Ali, R., Ritter, A. y Cooke. R. (2005). The characteristics of heroin users entering treatment: Findings from the Australian treatment outcome study (ATOS). Drug and Alcohol Review, 24, 411-418. doi:10.1080/09595230500286039.

Sáiz, P. y Bobes, J. (2014). Suicide prevention in Spain: An uncovered clinical need. Revista de Psiquiatría y Salud Mental, 7, 1-4. doi:10.1016/j.rpsm.2014.01.003.

Tiet, Q. Q., Ilgen, M. A., Byrnes, H. F. y Moos, R. H. (2006). Suicide attempts among substance use disorder patients: An initial step toward a decision tree for suicide management. Alcoholism: Clinical and Experimental Research, 30, 998-1005. doi:10.1111/j.1530-0277.2006.00114.x.

Voss, W. D., Kaufman, E., O’Connor, S. S., Comtois, K. A., Conner, K. R. y Ries, R. K. (2013). Preventing addiction related suicide: A pilot study. Journal of Substance Abuse Treatment, 44, 565-569. doi:10.1016/j.jsat.2012.10.006.

Wang, L. J., Huang, Y. C., Lee, S. Y., Wu, Y. W. y Chen, C. K. (2014). Switching suicide methods as a predictor of completed suicide in individuals with repeated selfharm: A community cohort study in northern Taiwan. Australian $\mathcal{E}$ New Zealand Journal of Psychiatry, 49, 65-73. doi:10.1177/0004867414553951.

Wasserman, D., Iosue, M., Wuestefeld, A. y Carli, V. (2020). Adaptation of evidence-based suicide prevention strategies during and after the COVID-19 pandemic. World Psychiatry, 19, 294-306. doi:10.1002/wps.20801.

Wilcox, H. C., Conner, K. R. y Caine, E. D. (2004). Association of alcohol and drug use disorders and comple- ted suicide: An empirical review of cohort studies. Drug and Alcohol Dependence, 76, 11-19. doi:10.1016/j.drugalcdep.2004.08.003.

World Report on Violence and Health. (2002) (Geneva: WHO). Recuperado de http://whqlibdoc.who.int/publications/2002/ 9241545615chap7eng.

Yuodelis-Flores, C. y Ries, R. K. (2015). Addiction and suicide: A review. American Journal on Addictions, 24, 98-104. doi:10.1111/ajad.12185.

Zalsman, G., Hawton, K., Wasserman, D., Heeringen, K. Van, Arensman, E., Sarchiapone, M.,... Zohar, J. (2016). Suicide prevention strategies revisited: 10-year systematic. Lancet Psychiatry, 3, 646-659. doi:10.1016/S22150366(16)30030-X.

Zalsman, G., Hawton, K., Wasserman, D., Heeringen, K. Van, Arensman, E., Sarchiapone, M.,... Zohar, J. (2017). Evidence-based national suicide prevention taskforce in Europe: A consensus position paper. European Neuropsychopharmacology, 27, 418-421. doi:10.1016/j.euroneuro.2017.01.012. 
